\begin{tabular}{|c|c|}
\hline Title & Preparation of Tough Double and Triple Network Supermacroporous Hydrogels through Repeated Cryogelation \\
\hline Author(s) & $\begin{array}{l}\text { Sedla ík, Tomán; Nonoyama, T akay uki; Guo, Honglei; Kiyama, Ryuji; Nakajima, T asuku; T akeda, Y oshihiro; } \\
\text { Kurokawa, Takayuki; Gong, Jian Ping }\end{array}$ \\
\hline Citation & $\begin{array}{l}\text { Chemistry of Materials, 32(19), 8576-8586 } \\
\text { https://doi.org/10.1021/acs.chemmater.0c02911 }\end{array}$ \\
\hline Issue Date & $2020-10-13$ \\
\hline Doc URL & http:/hdl .handle.net/2115/82930 \\
\hline Rights & $\begin{array}{l}\text { This document is the A ccepted Manuscript version of a Published Work that appeared in final form in Chemistry of } \\
\text { Material s, copyright c A merican Chemical Society after peer review and technical editing by the publi isher. To access } \\
\text { the final edited and published work see http://pubs.acs.org/.10.1021/acs.chemmater.0c02911. }\end{array}$ \\
\hline Type & article (author version) \\
\hline Additional Information & There are other files related to this item in HUSCAP. Check the above URL. \\
\hline File Information & Chemistry of Materials_2020.pdf \\
\hline
\end{tabular}

Instructions for use 


\title{
Preparation of Tough Double- and Triple-Network Supermacroporous Hydrogels through Repeated Cryogelation
}

\author{
Tomáš Sedlačíkª,b, Takayuki Nonoyamaa,b, Honglei Guo a,b, Ryuji Kiyamac, Tasuku Nakajimaa,b,d, Yo- \\ shihiro Takedae, Takayuki Kurokawa,b, Jian Ping Gonga,b,c,* \\ aFaculty of Advanced Life Science, Hokkaido University, Kita-21 Nishi-11, Kita-ku, Sapporo, 001-0021, Japan \\ bGlobal Station for Soft Matter (GI-CoRE), Hokkaido University, Kita-21 Nishi-11, Kita-ku, Sapporo, 001-0021, Japan \\ 'Graduate School of Life Science, Hokkaido University, Kita-21 Nishi-11, Kita-ku, Sapporo, 001-0021, Japan \\ dInstitute for Chemical Reaction Design and Discovery (WPI-ICReDD), Kita-21 Nishi-11, Kita-ku, Hokkaido University, \\ Sapporo 001-0021, Japan
}

eRigaku Corporation, 3-9-12 Matsubara-cho, Akishima-shi, Tokyo 196-8666, Japan

\begin{abstract}
Supermacroporous hydrogels, possessing sponge-like structure and permeability, have drawn significant attention for their bioengineering and biomedical applications. However, their mechanical weakness due to their low-density structure is one of their biggest limitations. This work reports a multi-step cryogelation technique, which does not require special equipment, for preparing tough supermacroporous hydrogels on the basis of the double-network (DN) strategy. The produced supermacroporous DN gels possess interconnected pores with pore sizes of 50-230 $\mu \mathrm{m}$. They also show a compressive modulus of up to $\sim 100 \mathrm{kPa}$, which is 2- to 4-times higher than that of the corresponding supermacroporous singlenetwork (SN) gels, and compressive strength of up to $1 \mathrm{MPa}$ at $80 \%$ compression. The supermacroporous DN cryogels are also stretchable with a work of extension of up to $38 \mathrm{~kJ} \mathrm{~m}^{-3}$, which is 1 to 2 orders larger than that of the SN cryogels. The high stiffness and stretchability distinguish them from other types of cryogels. Supermacroporous triple-network (TN) gels and DN gels composed of different polymer combinations are also prepared. The technique presented herein is suitable for preparing supermacroporous DN gels from various polymers; hence, it is promising in meeting bioengineering and biomedical demands.
\end{abstract}

\section{Introduction}

Hydrogels with pores larger than $1 \mu \mathrm{m}$ are called supermacroporous hydrogels to distinguish them from the macroporous polymer networks with pores ranging from $50 \mathrm{~nm}$ to $1 \mu \mathrm{m}$ as defined by International Union of Pure and Applied Chemistry (IUPAC). ${ }^{1,2}$ This work focused on supermacroporous hydrogels with interconnected pores larger than human cells, i.e., larger than $10 \mu \mathrm{m}$. Due to their sponge-like structure, such supermacroporous hydrogels are permeable to large particles such as cells, while possessing the biological and mechanical compatibility to biological tissues. ${ }^{1}$ Supermacroporous hydrogels are desirable materials in many applications, especially in bioengineering and biomedicine. For example, they are used in three-dimensional dynamic cell cultivation, bio-separation, or tissue engineering. For tissue engineering applications, soft and biocompatible scaffolds with sufficiently large interconnected pores are specifically required to allow cell migration and proliferation. ${ }^{3,4}$

However, most of the existing supermacroporous hydrogels are mechanically weak; this has limited their potential applications. This mechanical weakness is mainly because of two reasons. First, the mechanical strength of a material decreases with the increase in the volume fraction of pores. $^{5,6}$ The pores are considered as defects in the bulk materials, through which cracks are initiated. ${ }^{7}$ Second, the supermacroporous hydrogels are usually made of singlenetwork (SN) hydrogels that are intrinsically weak materials because of the lack of energy dissipation mechanisms. ${ }^{8,9}$ Accordingly, a straightforward strategy to improve the mechanical strength of porous hydrogels is to use high strength hydrogels together with a suitable technique.

Since 2000, efforts have been devoted toward improving the mechanical strength and toughness of gels. ${ }^{10-15}$ In 2003, the double-network (DN) strategy for preparing tough gels was introduced and has been intensively researched since. ${ }^{10,16-19}$ DN gels are a subcategory of interpenetrating polymer network (IPN) gels consisting of two contrasting networks. The first network is stiff and brittle; hence, it provides sacrificial bonds to dissipate mechanical energy upon excessive mechanical loading of the DN gel, while the second network is soft and ductile, thereby allowing effective distribution of the mechanical energy within the DN gel. This contrasting structure ensures that the $1^{\text {st }}$ network breaks only into multiple small fragments connected by the $2^{\text {nd }}$ network, thereby preventing the early global fracture of the material. Despite their high water content ( 90 wt.\%), DN gels, with an optimized structure, possess excellent hardness (elastic modulus of 0.1-1.0 MPa), strength (tensile failure stress 1-10 MPa, compressive failure stress 20-60 
$\mathrm{MPa}$ ), and deformability (failure tensile strain 1000-2000\%, failure compressive strain $90-95 \%){ }^{16,17}$ Moreover, they also exhibit extremely high toughness, i.e., high resistance to crack growth $\left(10^{2}-10^{4} \mathrm{~J} \mathrm{~m}^{-2}\right)$, that is two orders higher than that of typical SN gels $\left(10^{0}-10^{1} \mathrm{~J} \mathrm{~m}^{-2}\right) \cdot{ }^{16,17}$ These unprecedented mechanical properties are comparable to or even exceed some soft load-bearing natural tissues. ${ }^{18,19}$

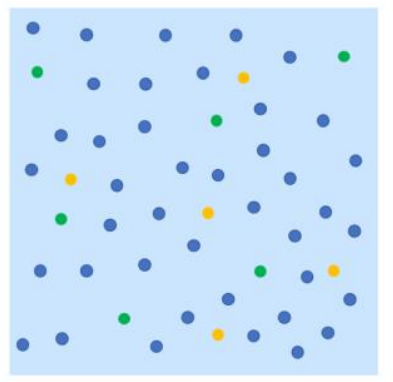

$1^{\text {st }}$ pre-gel solution

(NaAMPS, MBAAm, initiator)

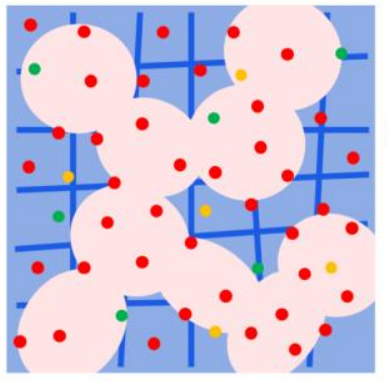

$1^{\text {st }} \mathrm{gel}$ in $2^{\text {nd }}$ pre-gel solution (AAm, MBAAm, UV initiator)

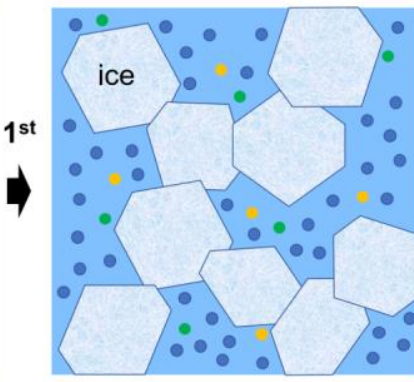

frozen pre-gel solution

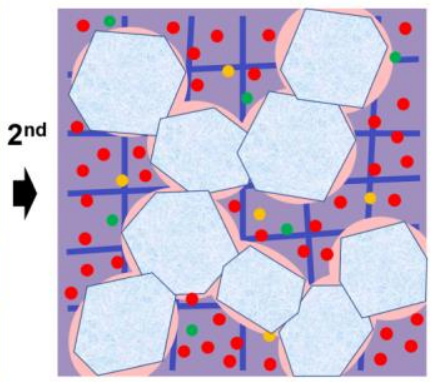

frozen

$1^{\text {st }}$ gel in $2^{\text {nd }}$ pre-gel solution

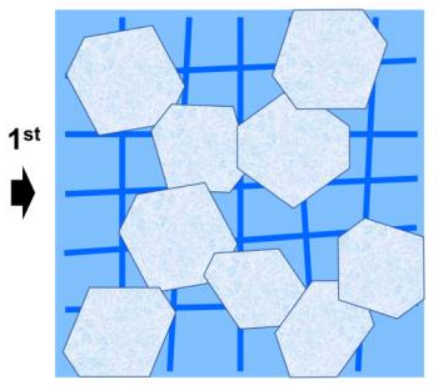

polymerization $\rightarrow$ frozen supermacroporous SN gel

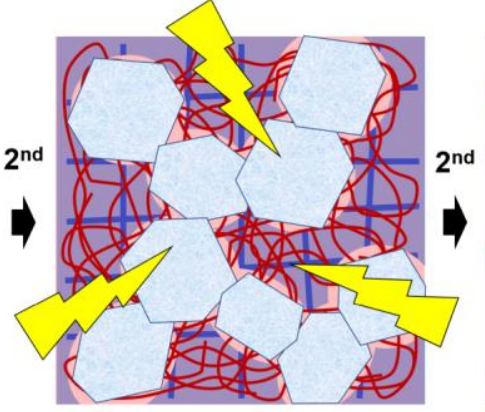

UV polymerization $\rightarrow$ frozen supermacroporous $\mathrm{DN}$ gel

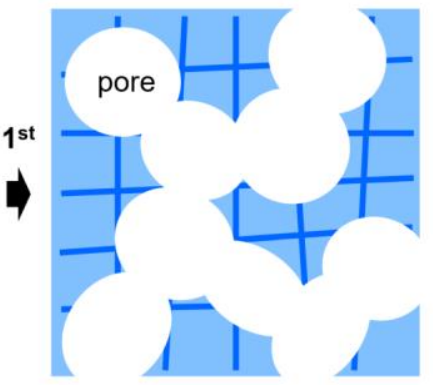

defrosted supermacroporous $\mathrm{SN}$ gel ( $\left.1^{\text {st }} \mathrm{gel}\right)$

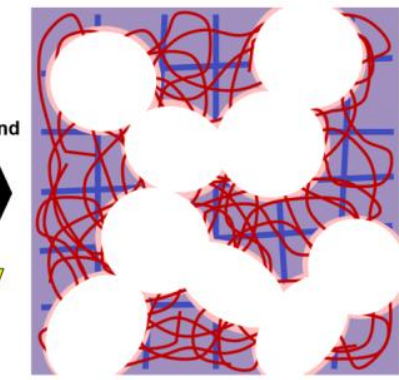

defrosted supermacroporous DN gel ( $\left.2^{\text {nd }} \mathrm{gel}\right)$

Scheme 1. Schematic representation of the two-step cryogelation used to prepare supermacroporous DN gels. First, the supermacroporous $S N$ gel $\left(1^{\text {st }}\right.$ gel) was synthesized by $1^{\text {st }}$ cryogelation, that is cross-linking polymerization in the frozen state. Then, the $1^{\text {st }}$ gel was swollen in the $2^{\text {nd }}$ pre-gel monomer solution that contained a UV initiator and frozen again. Since the ice crystallization of $2^{\text {nd }}$ network solution occurred preferentially inside pores of the $1^{\text {st }}$ network gel, the $1^{\text {st }}$ network gel acted as a template for the formation of ice crystals. As a result, the $1^{\text {st }}$ gel supermacroporous morphology was preserved during the $2^{\text {nd }}$ polymerization that was initiated by UV irradiation of the frozen sample.

Therefore, supermacroporous hydrogels derived from DN gels could exhibit better mechanical properties than the conventional supermacroporous hydrogels made from SNs or even other IPNs. Furthermore, because the DN effect only requires a contrasting DN structure and is independent of the chemical species of polymers, the synthesis approach, once established, can also be applied to various polymers in demand, such as biocompatible polymers. Notably, this approach can assist in the preparation of DN hydrogels with an open-cell morphology, which allows the proliferation of cells, as it is typically challenging. Recently, Kovačič et al. prepared supermacroporous DN gels using a sequential solvent casting technique. ${ }^{20}$ Although the pores of the gels made by this method were several tens of micrometers in diameter, interconnections among the pores were small for cell migration and proliferation.

Supermacroporous gels are usually prepared by several methods, including particle leaching, solvent casting, 3Dprinting processes, gas foaming, freeze-drying, and cryogelation. ${ }^{8}$ Among them, cryogelation is potentially the most promising technique since it does not require special equipment or use of toxic solvents. Cryogelation involves crosslinking polymerization in the presence of solvent crystalsusually ice-as porogens, that forms sponge-like gels with highly interconnected pores. ${ }^{21,22}$ At certain temperatures below the solvent freezing point, the solvent of the gel precursor solution forms crystals, while the gel precursors, that are concentrated in the interstitial spaces between solvent crystals, form a gel by gelation. The pore size of cryogels is determined by several factors such as freezing temperature and rate, the concentration of gel precursors and initiator, if any, and size and shape of sample mold. These cryogels have been widely studied for various biomedical applications. ${ }^{1,23-}$ 25

Attempts to prepare cryogels with enhanced mechanical properties, such as hybrid cryogels from two inter-crosslinked polymers, IPNs, and even DNs, have also been made. 26-37 However, these previous studies on porous cryogels hardly showed improved mechanical properties, since the contrasting network structure required for the DN effect 
was not formed in these cryogels. Furthermore, multi-step gelation can result in disconnected pore structures. ${ }^{27}$ This disconnected pore structure possibly arises because of the use of polymeric precursors instead of monomers, because the $2^{\text {nd }}$ gelation proceeds faster than freezing and so the $2^{\text {nd }}$ gelation occurs in pores, or because monomers did not diffuse sufficiently into the $1^{\text {st }}$ network phase. In this work, we established a two-step cryogelation method to overcome these issues and to synthesize supermacroporous DN hydrogels with high mechanical toughness and an interconnected pore structure. Based on the DN theory, we adopted highly swollen polyelectrolyte as the $1^{\text {st }}$ network and neutral polymer as the $2^{\text {nd }}$ network. These networks were combined using a two-step polymerization process to form the contrasting double-network structure. To preserve the interconnected porous morphology formed by the first step cryogelation, we controlled the ice crystal formation in the second step. Then we used UV initiated polymerization so that the $2^{\text {nd }}$ network formed in the gel phase of the $1^{\text {st }}$ network, instead of in the pores (Scheme 1). We thus fabricated the supermacroporous hydrogels using conventional DN systems, made from polyelectrolyte, poly(2-acrylamido2-methylpropane sulfonic acid, sodium salt) (PNaAMPS), as the rigid $1^{\text {st }}$ network and neutral polymers, poly(acrylamide) (PAAm), poly(2-hydroxyethyl methacrylate) (PHEMA), and poly( $N, N$-dimethylacrylamide) (PDMAAm), as the flexible $2^{\text {nd }}$ network. The porous structure and mechanical properties of these DN cryogels were characterized.

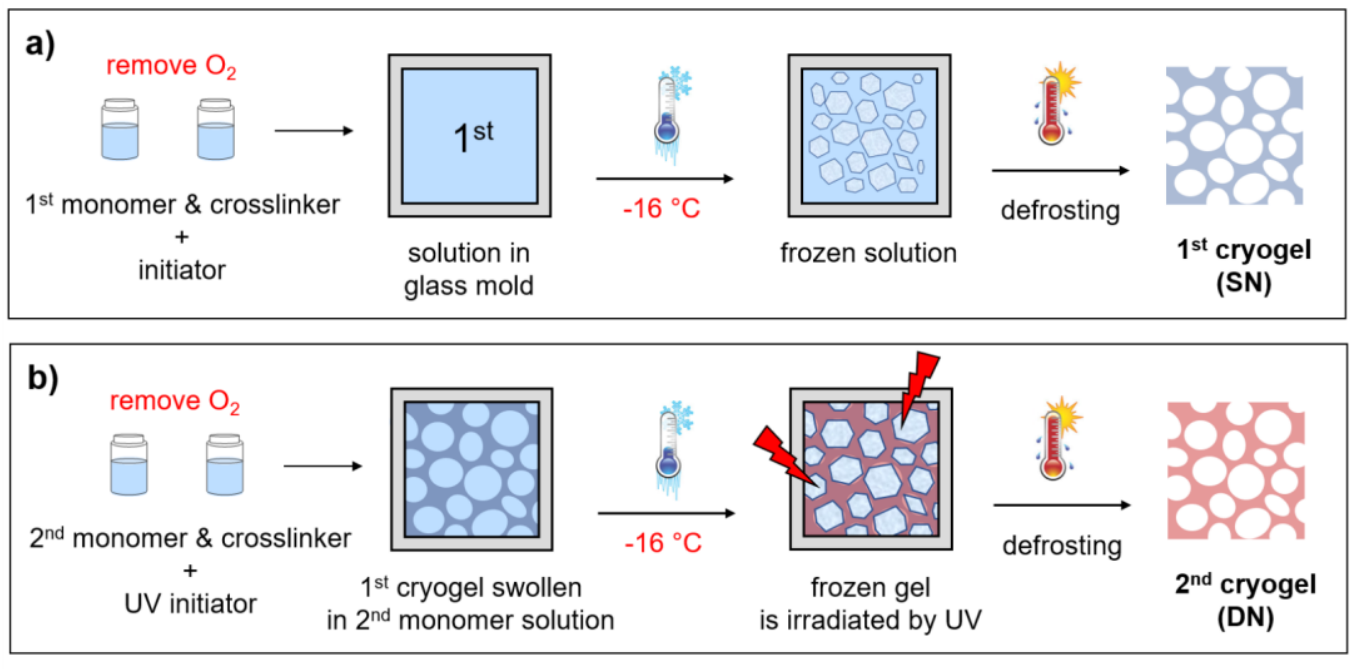

d) c)

$1^{\text {st }}$ network monomer:

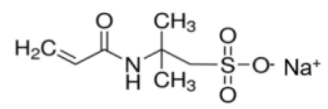
NaAMPS

$2^{\text {nd }}$ network monomer:<smiles>C=CC(N)=O</smiles>

AAm<smiles>C=CC(=O)NCNC(=O)COC(C)(C)C</smiles>

Scheme 2. Schematic representation of the DN cryogels two-step preparation, respective monomer structures and sample coding. Firstly, SN cryogels are prepared (a): The monomer solution of the $1^{\text {st }}$ network is mixed with a solution of red-ox initiator, and the reaction mixture is transferred into a mold consisting of two glass plates with a silicone spacer. Then, the mold is placed into a cooling thermostat to freeze and polymerize. After that, samples are defrosted, and SN cryogels are removed from the mold and swollen in water to equilibrium; Secondly, DN cryogels are prepared (b): The SN cryogel is swollen in a monomer solution of the $2^{\text {nd }}$ network with a UV initiator, and then the sample is placed into the mold and frozen for $3 \mathrm{~h}$ to allow for the diffusion of reactants into the $\mathrm{SN}$ gel phase. After that, the frozen sample is UV irradiated to promote $2^{\text {nd }}$ gelation; Since the process is based on radical polymerization, oxygen should be removed in both steps. The molecular structures of the $1^{\text {st }}$ and $2^{\text {nd }}$ network monomers, as well as the crosslinker, are shown in (c); Sample coding is explained in (d).

\section{Experimental}

\subsection{Materials}

Acrylamide (AAm) (Extra pure, $\geq 98.0 \%$ ) was purchased from Junsei Chemical Co. Ltd and was purified to remove any inhibitors by recrystallization from chloroform $(100 \mathrm{~g} /$ $300 \mathrm{~mL}, 5^{\circ} \mathrm{C}$, with a yield of $\left.\sim 90 \mathrm{wt} . \%\right) . N, N$-dimethylacrylamide (DMAAm), cyclohexane $\left(1^{\text {st }}\right.$ grade $\left.>98 \%\right)$, lead(II) nitrate (99.5+\%), $N, N^{\prime}$-methylenebis(acrylamide) (MBAAm, $\geq$ $99 \%$ for electrophoresis), $N, N, N^{\prime}, N^{\prime}$-tetramethylenediamine
(TEMED, $\geq 99 \%$ for electrophoresis), 2-oxoglutaric acid (2oxo, $>99 \%)$, and sodium chloride $\left(\mathrm{NaCl}, \geq 99.51^{\text {st }}\right.$ grade) were purchased from Fujifilm Wako Pure Chemical Cooperation, Japan. DMAAm was then redistilled under vacuum. 2acrylamido-2-methylpropane sulfonic acid, sodium salt (NaAMPS, 49.7 wt.\% solution) was purchased from Toagosei Co., Ltd., Japan. Ammonium persulfate (APS, $\geq$ $98 \%$ for electrophoresis, tested for use in acrylamide polymerization), 2-hydroxyethyl methacrylate (HEMA, $\geq$ 
$99 \%$, with $\leq 50$ ppm monomethyl ether hydroquinone as an inhibitor) and fluorescein $o$-acrylate (95\%) were purchased from Sigma-Aldrich, US. Argon gas (99.999\%; $\mathrm{O}_{2}<0.2 \mathrm{ppm}$ ) as inert gas for radical polymerization was purchased from Air Water Inc., Japan. Milli-Q ${ }^{\circledR}$ water (model Integral 5, from Merck Millipore, US) was exclusively used in all experiments. Ethanol ( $\geq 99.5 \%$ ) was purchased from Fujifilm Wako Pure Chemical Cooperation, Japan.

\subsection{Preparation of SN cryogels}

During the preparation of supermacroporous cryogels with an interconnective pore structure, polymerization must occur after freezing or be slower than freezing. ${ }^{38} \mathrm{How}$ ever, it is also known that proceeding polymerization helps the system to freeze. We thus decided to use a red-ox initiation system (APS with TEMED) to prepare the SN cryogels based on the PNaAMPS polyelectrolyte network. In that case, polymerization can be set to be slower than freezing and, in our opinion, samples freeze more easily and uniformly than if UV initiators were used (no polymerization prior irradiation). The concentration of reactants triggered by freezing allows for the preparation of gels from diluted monomer solutions. The preparation procedure of SN cryogels is depicted in Scheme 2. First, a monomer solution of prescribed amounts of NaAMPS, MBAAm, and TEMED (typically for preparation of SN cryogel 10-4: [4.35 g of NaAMPS (49.7 vol.\%), $0.06 \mathrm{~g} \mathrm{MBAAm,} 5.5 \mathrm{~mL}$ water] $4.5 \mathrm{~mL}+$ water $4.5 \mathrm{~mL}+\left[10 \mathrm{mg} \mathrm{mL}^{-1}\right.$ TEMED] $0.5 \mathrm{~mL}$ ) was purged with argon for $20 \mathrm{~min}$ to remove any dissolved oxygen, that is a radical polymerization inhibitor. Then, a solution of APS (10 $\mathrm{mg} \mathrm{mL}^{-1}$ ), also purged by argon, was added to the monomer solution ( $0.5 \mathrm{~mL}$ to $9 \mathrm{~mL}$ ). The solutions were precooled in an ice bath before mixing to slow down the polymerization process. A glass mold, with inner dimensions $60 \times 60 \times 2$ $\mathrm{mm}$, was constructed from two rectangular glass pieces with a silicone spacer and stopper. This mold was precooled at $-16^{\circ} \mathrm{C}$ in an ethanol cooling bath (NCB-3300, Tokyo Rikakikai Co, Ltd, Japan) before injecting the reaction solution. The mold with injected reaction solution was then sealed in a plastic bag and immediately placed in the ethanol cooling bath. The frozen sample was kept at $-16^{\circ} \mathrm{C}$ overnight to promote high yield cross-linking polymerization. After polymerization, the mold was taken out of the cooling bath and gently defrosted under cold tap water. The brittle asprepared gel was then soaked in ethanol to avoid potential damage by rapid swelling in water. Next, the gel was placed in deionized water to remove any unreacted monomers and soluble products of radical polymerization and equilibrate. Bath water was frequently changed for several days. When the dialysate conductivity, measured by a conductometer (FiveEasy F30, Mettler-Toledo Inc., US), reached pure water conductivity (approx. $1.5 \mu \mathrm{S} \mathrm{cm}^{-1}$ ), dialysis was considered to be finished.

SN cryogels were coded as $M_{1}-X_{1}$, where $M_{1}$ is the total monomer concentration ( $\mathrm{mol} \mathrm{L}^{-1}$ ) including the crosslinker and $x_{1}$ is the molar ratio of the crosslinker MBAAm relative to the monomer NaAMPS (mol\%) in the gel precursor solution.

\subsection{Preparation of DN and TN cryogels}

The $2^{\text {nd }}$ network was synthesized by UV initiated polymerization after freezing the SN cryogel that was first equilibrated in water and then pre-soaked in a $2^{\text {nd }}$ monomer solution. The polymerization and freezing rates were again important. On the one hand, if the sample freezing rate were too fast, smaller crystals would be formed in the original SN cryogel macropores; this would noticeably affect the resulting DN gel morphology. Hence, the $2^{\text {nd }}$ cryogelation temperature should not be lower than the $1^{\text {st }}$ cryogelation temperature. We kept cryogelation temperature equal in both steps, that is $-16^{\circ} \mathrm{C}$. On the other hand, the original morphology of swollen SN could be significantly affected by the formation of $2^{\text {nd }}$ gel phase inside pores, if the $2^{\text {nd }}$ network polymerization was faster than the freezing. However, this issue was solved by using photo-initiators. It ensured that polymerization could be initiated after the sample was frozen and ice crystals filled the pores. To ensure the formation of the DN phase, samples were irradiated with UV 3 hours after being frozen, so that the monomers and initiators had enough time to diffuse into the SN gel phase. The preparation procedure of DN cryogels is depicted in Scheme 2. The SN cryogel sheet was dialyzed against water and cut into cylindrical samples of $\sim 35 \mathrm{~mm}$ in diameter. The edges of the SN cryogel sheet were cut off $(\sim 1 \mathrm{~cm}$ from each side $)$ to prepare the DN cryogel sheet. The SN cryogel sample was placed in a container of appropriate dimensions (just a little larger than the sample) and transferred into a glove box, where the sample was kept under inert argon atmosphere overnight to remove dissolved oxygen. The sample was covered in a loosely closed plastic dish to avoid gel drying. If the sample partly shrunk, water was replenished, while any excess water was aspirated by pipetting. Meanwhile, the three solutions of respective reactants for the $2^{\text {nd }}$ network formation were purged with argon for 20 min (stock solution of monomers from $2.22 \mathrm{~g}$ AAm and $4.8 \mathrm{mg}$ MBAAm dissolved in $6.78 \mathrm{~mL}$ water; solution of TEMED: $25 \mathrm{mg} \mathrm{mL}^{-1}$, and solution of 2-oxo: $10 \mathrm{mg} \mathrm{mL}^{-1}$ ), and then mixed at a volume ratio of $9: 0.5: 0.5$, that gave a monomer concentration of $2.8 \mathrm{~mol} \mathrm{~L}^{-1}$ ( 20 wt.\%). The reaction mixture volume added to the SN cryogel was equal to the sample volume as determined by weighing (the density of the porous SN gel was approx. $1.01 \mathrm{~g} \mathrm{~cm}^{-3}$ ); this gave a final monomer concentration of $1.4 \mathrm{~mol} \mathrm{~L}^{-1}$ (dilution factor $=2$ ). However, to get a lower final monomer concentration, the stock monomer solution was first diluted by water, so the TEMED (and 2-oxo) concentration remained constant for all samples. The SN cryogel in the mixture of reactants was covered with an alumina foil to avoid unwanted photopolymerization. After equilibration in a glove box for $3 \mathrm{~h}$ at $40 \mathrm{rpm}$, the gel was placed between two glasses with a silicone spacer of appropriate thickness and moved from the glove box into the cooling bath $\left(-16^{\circ} \mathrm{C}\right)$ where samples were kept $3 \mathrm{~h}$ prior UV irradiation. During the cooling process, the ice crystals were formed in the pores of the first SN cryogel, while the second monomers and initiators were concentrated in the SN gel phase.

To maintain the original morphology of SN cryogel, the $2^{\text {nd }}$ cryogelation was performed using 2 -oxo and TEMED for 
photo-initiation. The $2^{\text {nd }}$ network UV polymerization was performed using a UV-light-emitting diode irradiation panel (UAW365-31110-1212F, Sentech Co.) in a custom safety box. Samples were removed from the cooling bath and irradiated in short pulses ( $5 \mathrm{~s}$ from each side, 4 cycles, $40 \mathrm{~s}$ in total) to avoid melting them by the heat that was generated by the UV panel ( $\sim 25 \mathrm{~mm}$ distance, $\left.\sim 125 \mathrm{~mW} \mathrm{~cm}^{-2}\right)$. This irradiation duration did not cause sample melting to a visible extent. For the same reason, samples were immersed in the cooling bath for $\sim 5$ min before the next irradiation cycle. We realized that an excess addition of TEMED to 2-oxo was essential to obtain reproducible results when using highpower UV irradiation with short pulses. Without TEMED, we observed a certain extent of gel softening in comparison to the original SN cryogels. The high-power UV technique generates many radicals during a short time. These primary radicals undergo recoupling, and thus polymerization takes place randomly at a low yield, resulting in short polymer chains that soften the $1^{\text {st }}$ network. We assumed that TEMED, as a donor of an unpaired electron, could stabilize the primary radicals and promote the 2-oxo decomposition upon UV irradiation. The TEMED excess was probably necessary because 2-oxo is a protonic acid and the protonated amine co-initiator does not initiate polymerization. ${ }^{39}$ It is worth to note that similar results were achieved by using a common low-power UV-lamp (365 nm) placed over the cooling bath with a glass cover. In such a case, TEMED addition was not necessary, and samples were placed horizontally in the cooling bath and irradiated overnight (with safety shielding). Therefore, DN cryogels can be easily prepared in any laboratory without special equipment. After irradiation was completed, the samples were immersed in the cooling bath $\left(-16^{\circ} \mathrm{C}\right)$ for $20 \mathrm{~h}$ to allow polymerization to high yield. After polymerization, the sample was gently defrosted under cold tap water while in the mold. Finally, the gel was rinsed and equilibrated in pure water for several days with frequent water exchange.

DN cryogels were coded as $M_{1}-X_{1} / M_{2}-X_{2}$, where $M_{1}$ describes the monomer concentration $\left(\mathrm{mol} \mathrm{L}^{-1}\right)$ including the crosslinker and $x_{1}$ is the molar ratio of the crosslinker relative to the monomer (mol\%) used for the $1^{\text {st }}$ network preparation, $M_{2}$ and $x_{2}$ have the same meaning but indicate the parameters for the $2^{\text {nd }}$ network. TN cryogels were prepared from DN cryogels by a similar procedure and were marked as $M_{1}-X_{1} / M_{2}-X_{2} / M_{3}-X_{3}$, where $M_{3}-X_{3}$ describes the $3^{\text {rd }}$ network composition.

\subsection{Characterization of SN, DN and TN cryogels}

\subsubsection{Swelling and porosity}

The swollen cryogel was weighed to obtain its weight in the equilibrium swollen state $\left(m_{s w}\right)$, that is the weight of porous hydrogel with water in its pores. The sample was freeze-dried and weighed again to obtain the dry weight $\left(m_{d}\right)$. The equilibrium water regain (ratio $\left.Q_{w}\right)$ was calculated according to the following equation:

$$
Q_{w}=\frac{m_{s w}-m_{d}}{m_{d}}=\frac{m_{s w}}{m_{d}}-1
$$

The freeze-dried cryogel was then soaked in cyclohexane and quickly weighed to obtain the weight of the sample in a non-solvent $\left(m_{s w C H}\right)$. The porosity $\left(\mathrm{P}_{\mathrm{C}} \mathrm{CH}\right)$ of the dry sample was calculated according to the following equation ${ }^{8}$ :

$$
P_{\% C H}=100 \cdot \frac{\left(m_{S w C H}-m_{d}\right) / \rho_{C H}}{m_{S w C H} / \rho_{S w C H}}=100 \cdot\left(1-\frac{m_{d}}{m_{S w C H}}\right)
$$

where $\rho_{C H}$ is the cyclohexane density and $\rho_{s w C H}$ is the density of the sample with cyclohexane $\left(\rho_{C H} \approx \rho_{S w C H}\right.$ when $P_{\% C H}>$ $90 \%)$. The cryogel porosity $\left(\mathrm{P}_{\% \mathrm{CH}}\right)$ represents the volume fraction (in \%) of pores in a freeze-dried sample.

The porosity in the swollen state $\left(P_{\%}\right)$ was estimated using an equation analogous to Equation 2 . This equation uses the sample weight of the equilibrium swollen state $\left(m_{s w}\right)$ and the sample weight after compressing the sample between tissue papers to squeeze out the water in its macropores $\left(m_{s q}\right)$ :

$$
P_{\%}=100 \cdot \frac{\left(m_{s w}-m_{s q}\right) / \rho_{H 2 O}}{m_{s w} / \rho_{s w}}=100 \cdot\left(1-\frac{m_{d}}{m_{s w}}\right)
$$

where $\rho_{\text {H2O }}$ is the density of water, and $\rho_{s w}$ is the density of the water-swollen sample $\left(\rho_{s w} \approx \rho_{H 20}\right)$. The supermacroporous sample was thoroughly squeezed three times between tissue papers and then immediately weighed.

The water content in the gel phase only was also estimated from the same gel squeezing experiment as follows:

$$
W_{\% G E L}=100 \cdot \frac{m_{s q}-m_{d}}{m_{s q}}=100 \cdot\left(1-\frac{m_{d}}{m_{s q}}\right)
$$

\subsubsection{Scanning electron microscopy (SEM)}

SEM measurement was conducted using a JSM-6010LA scanning electron microscope (JEOL Ltd., Japan). Samples were freeze-dried and sputter-coated with a gold/palladium mixture using an E-1010 ion sputter system (Hitachi, Japan) and analyzed at the acceleration voltage of $20 \mathrm{kV}$ using a detector of backscattered electrons (BSE). Shrinking of samples by freeze-drying was up to $10 \%$ in one-dimensional direction. Central and side parts of the samples were observed to confirm the uniformity of the morphology.

\subsubsection{Confocal Laser Scanning Microscopy (CLSM)}

To stain the $1^{\text {st }}$ network, SN cryogel $0.44-4$ was prepared with the addition of Rhodamine B into polymerization mixture and dialyzed first against acidified water $(\mathrm{pH} \sim 4)$ and then pure water. The Rhodamine-B-stained SN cryogel was used to prepare the DN cryogel. To stain the DN cryogel $2^{\text {nd }}$ network, a small amount of fluorescein $o$-acrylate was added to the $2^{\text {nd }}$ pre-gel solution. Fluorescein $o$-acrylate was dissolved directly into the $2^{\text {nd }}$ monomer solution up to the final concentration in the reaction solution of $5 \times 10^{-4} \mathrm{~mol} \mathrm{~L}$ 1 . The stained samples were kept in the dark before observation to avoid unnecessary photobleaching. The stained cryogels were then observed using a Nikon Confocal system A1Rsi equipped with a Ti-E inverted microscope with a motorized XY stage, piezo Z stage, and Perfect Focus System. Nikon Plan Apo VC 60×/1.4 oil objective and Nikon GFP-B filter were used. Data were processed with NIS-Elements from Nikon.

\subsubsection{Nano-tomography (nano-CT)}

For nano-CT measurement, the DN cryogel sample 0.44 4/0.7-0.1 was incubated in 1 wt.\% water solution of $\mathrm{PbNO}_{3}$ and then in water to remove the excess lead (II) ions from the gel phase and pores. Nano-CT scan was performed with 
an X-ray microscope CT nano3DX (Rigaku Corporation, Japan). A sample piece was put on a 5-axis stage and irradiated with X-rays. In this experiment, a $\mathrm{Cu}$ target $(\mathrm{CuK} \alpha 8.0$ $\mathrm{keV}$ ) was used to observe the fine network structures. The tube voltage and current were set at $40 \mathrm{kV}$ and $30 \mathrm{~mA}$, respectively. The field of CT scan view was $0.67 \times 0.67 \times 0.67$ $\mathrm{mm}$ and the effective voxel was set at 0.62 microns. The sample was rotated 180 degree and 600 projection images

a)

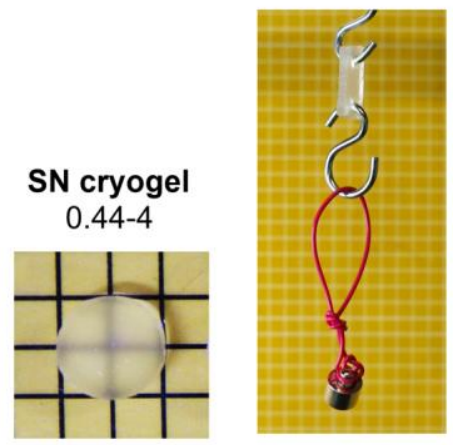

c)

DN cryogels

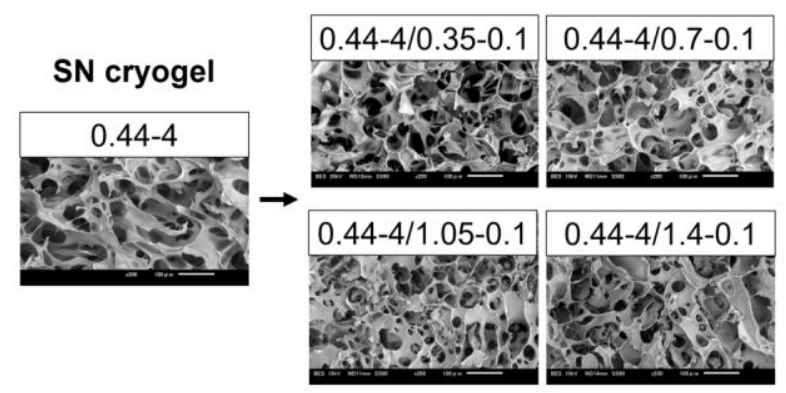

e)
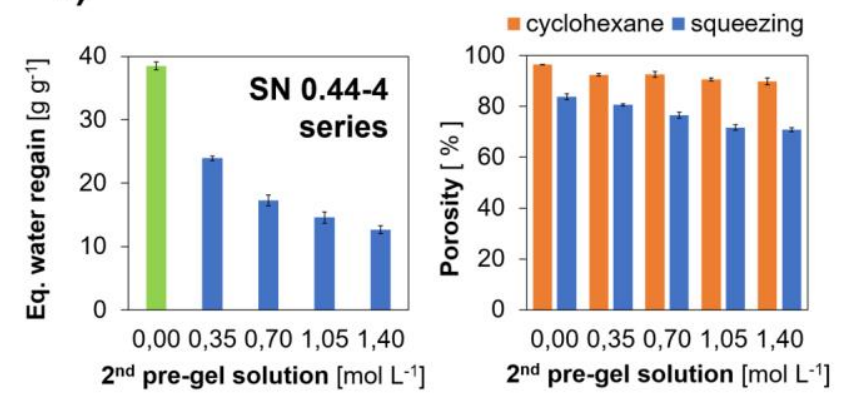

were captured. Then 1024 tomograms were reconstructed, and a 3D rendered image was visualized by Drishti $2.4 .^{40}$ The structures and pores were extracted and the three-dimensional thickness of structures was calculated by using Image J 1.50i with Local Thickness plugin. ${ }^{41,42}$ Then, the tomograms and 3D rendered image were recolored based on the thickness. The same approach was applied to the analysis of gel walls distance, referring to pore size.

b)

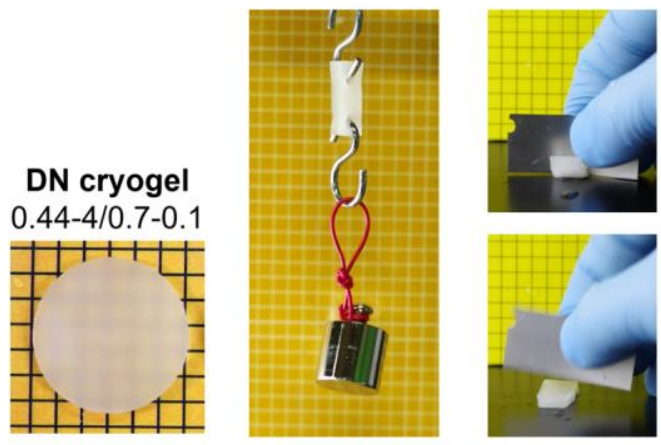

d)

DN cryogels

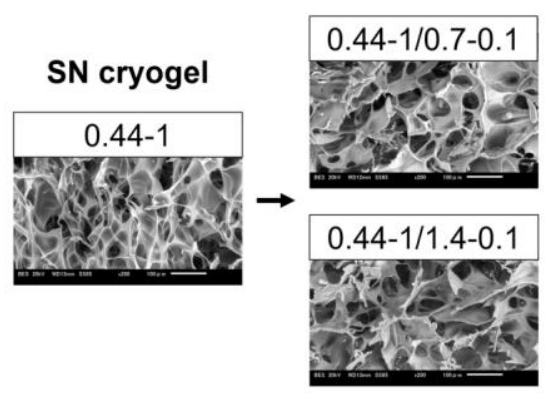

f)
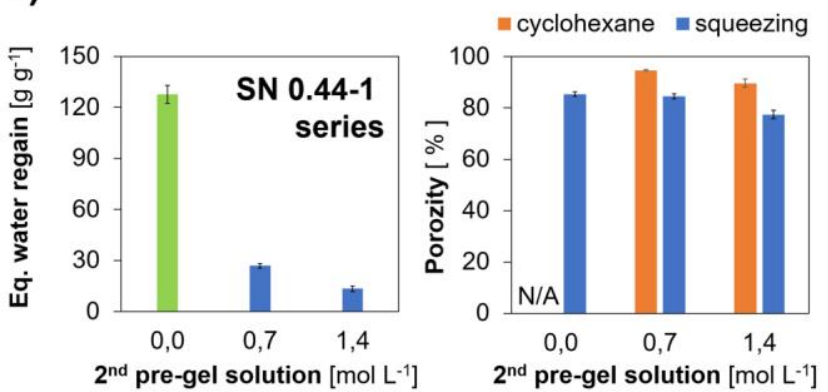

Figure 1. Visual appearance and first-sight mechanical performance of SN cryogel 0.44-4 (a) and selected DN cryogel 0.44-4/0.7-0.1 (b). SEM images of SN and DN cryogels showing their porous morphology (c, d) and equilibrium water regain and porosities (e, f) of two sets of samples (c, e: 0.44-4 series; d, f: 0.44-1 series). SN cryogel 0.44-4 was semi-transparent, unable to hold weights larger than 5 grams ( $\sim 3.0 \mathrm{~mm}$ thickness) and could easily be damaged (a). In contrast, the corresponding DN cryogel 0.44-4/0.7-0.1 ( $\sim 3.2$ $\mathrm{mm}$ thickness) was rather opaque, could be compressed to squeeze out water only with a large force, held weights as large as 50 grams, and resisted cutting (b). The scale bar in (c, d) is $100 \mu \mathrm{m}$.

\subsubsection{Compressive test}

The mechanical properties of the cryogels were investigated in compression using Tensilon RCT 1310A (Orientec, Japan). $100 \mathrm{~N}$ load cell was used to detect the stress response of the sample to the applied strain. At least three samples of each cryogel were investigated. For the compressive test, disc samples of $10 \mathrm{~mm}$ in diameter were cut from larger cryogel discs or rectangular sheets, that were swollen in water to equilibrium. The compression velocity was set constant as a $10 \%$ strain per minute $(\sim 0.25-$ $0.5 \mathrm{~mm} \mathrm{~min}^{-1}$ depending on the sample thickness given by the gel composition and respective degree of swelling). The 
correct initial gap size between the measuring plates, corresponding to full contact with the sample, was determined from a positive load when the loading force started to steep constantly. The compressive stress was estimated from the compressive force divided by the apparent sample surface area without deformation. All moduli presented in this work are nominal moduli, that are the moduli of the porous samples calculated from the slope at the beginning of the compressive stress-strain curve ( $\sim 0-5 \%$ compression).

\subsubsection{Tensile and cyclic tensile test of cryogels}

The mechanical properties of the cryogels were also investigated in tension using 1250A (Orientec, Japan). $100 \mathrm{~N}$ load cell was used to detect the stress response of the sample to the applied strain. At least three samples of each cryogel were investigated. For the tensile and tensile cyclic tests, the samples were cut by a Dumbbell shape cutter (12 $\mathrm{mm}$ length and $2 \mathrm{~mm}$ thickness). These cut samples were then tightly clamped (compressed) by clamps equipped with sandpaper to ensure that the samples do not slip during measurement. The measurement velocity was set to 10 $\mathrm{mm} \mathrm{min}^{-1}$. The nominal stress of the sample was calculated based on the apparent cross-sectional area of the porous sample before deformation ( $2 \mathrm{~mm} \times$ thickness of sample).

\section{Results and Discussion}

\subsection{Comparison of SN and DN cryogels}

SN cryogels based on PNaAMPS were prepared with a broad range of mechanical properties (Figure S1), from which samples $0.44-4$ and $0.44-1$ with compressive moduli of $29.1 \mathrm{kPa}$ and $3.5 \mathrm{kPa}$, respectively, were chosen for the preparation of DN cryogels (see Experimental section for sample coding). The SN cryogels were typically brittle, which is a prerequisite for toughening by introducing the ductile $2^{\text {nd }}$ network. Sample $0.44-4$ with 4 mol.\% of crosslinker was slightly opaque (Figure 1a), while sample 0.441 with $1 \mathrm{~mol} . \%$ of crosslinker was transparent. It is interesting that supermacroporous hydrogels were even transparent. The gel phase of such cryogels was highly swollen so that the refractive index of the gel phase was close to the refractive index of water. The (semi-)transparency of SN cryogels could ensure an advantage during the preparation of the DN cryogels as it allows even distribution of UV light within the sample during irradiation. Scanning electron microscopy (SEM) images of the SN cryogels of different composition are shown in Figure S2, showing similar morphology of interconnected pores $(\sim 50-100 \mu \mathrm{m})$. a)

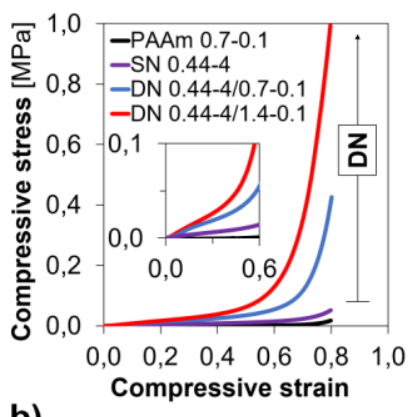

b)

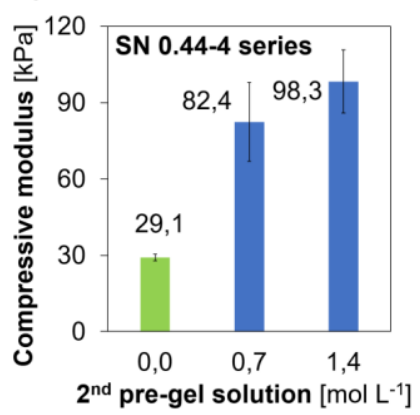

c)

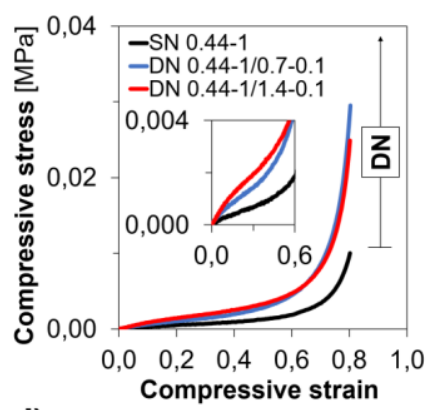

d)

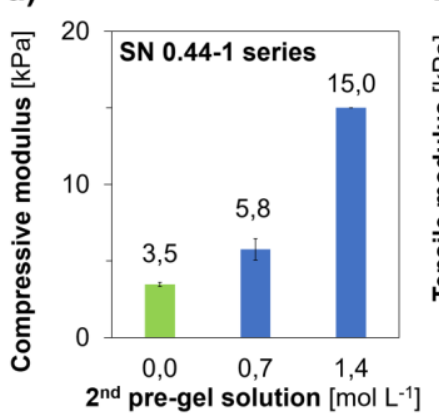

e)

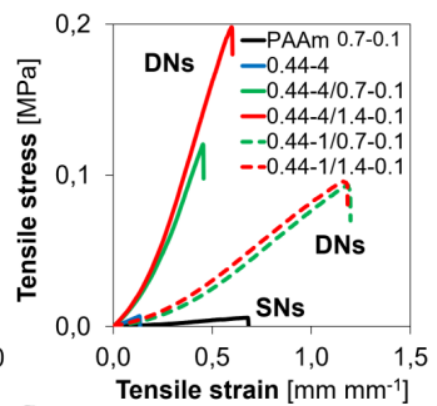

f)

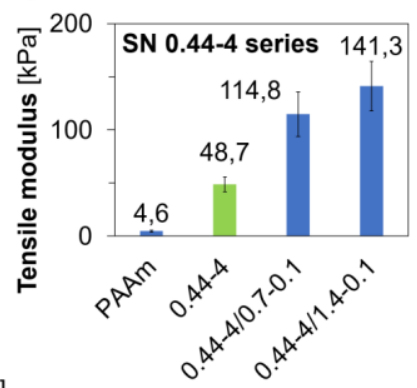

g)

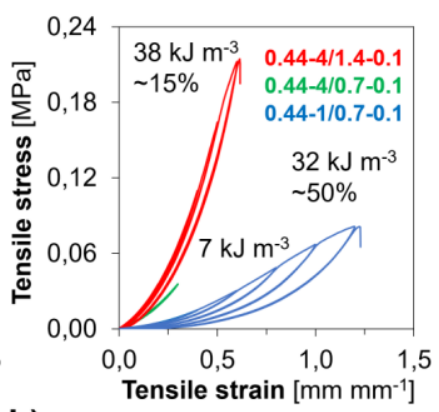

h)

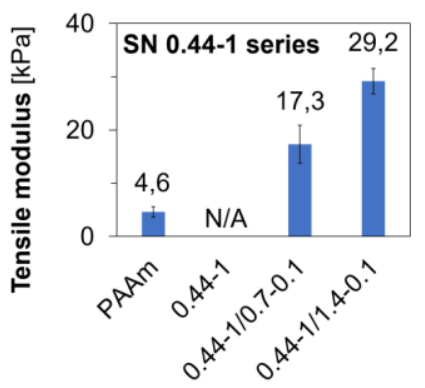

Figure 2. Mechanical properties of DN cryogels in comparison with their original SN cryogels measured under both compression (ad) and tension (e-h). The compressive test of SN cryogel 0.44-4 with 4 mol.\% of crosslinker (a), SN cryogel 0.44-1 with 1 mol.\% of crosslinker (c) and of their corresponding DN cryogels (a, c) and respective compressive moduli (b, d). DN cryogels were significantly stronger and stiffer than their original SN cryogels and pure $2^{\text {nd }}$ network PAAm cryogel. The tensile test of DN cryogels (e-g) showed remarkably improved tensile behavior in comparison with SN cryogels. DN cryogels showed hysteresis during the cyclic tensile test as typical for DN gels (g); the area of hysteresis loop (in \% of toughness) represents the ratio of mechanical energy dissipated by sacrificial bonding.

After the introduction of the PAAm $2^{\text {nd }}$ network, the cryogels became semi-transparent or opaque (Figure 1b). The higher concentration of the second monomer solution, the opaquer the porous DN gel was. While SN cryogels were easy to cut and required careful handling, DN cryogels could be bent or repeatedly compressed without apparent 
damage (Video S1). During compression, water in their pores drained out. After releasing compressive stress, the cryogels quickly retained their original shape, soaking water back due to capillary forces. This behavior indicated that

a)

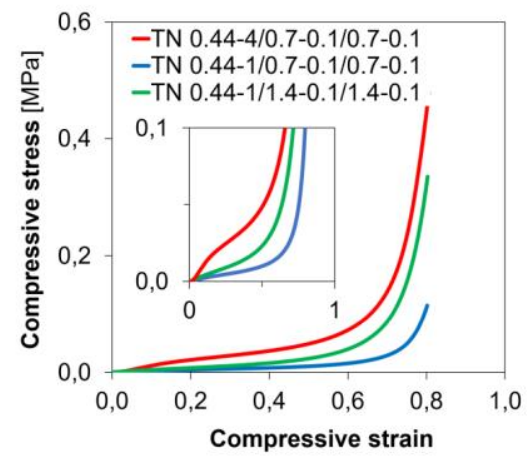

b)

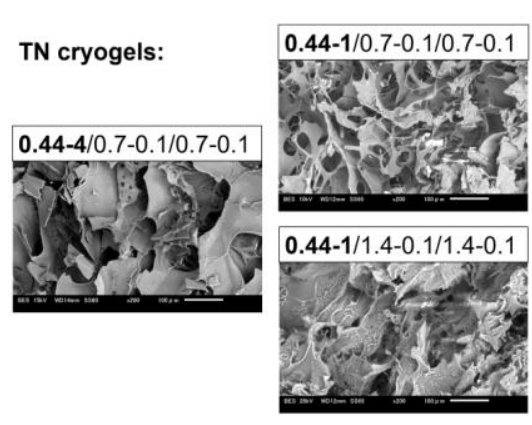

c)

d)
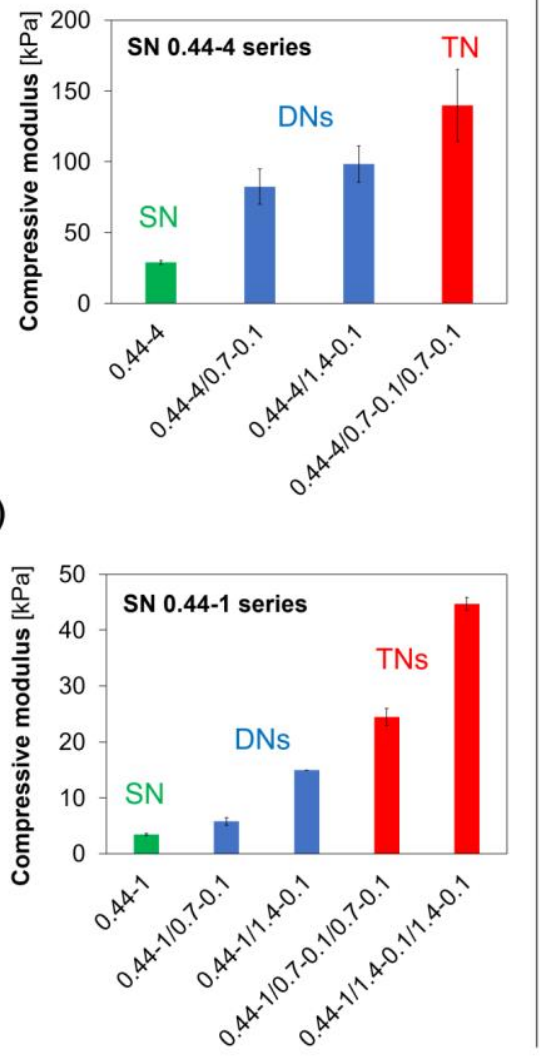

) the DN cryogels had interconnected macropores. Furthermore, DN cryogels could hold relatively large weights and even showed certain unusual cutting resistance (Figure $1 \mathrm{~b}$ with screenshots from SI video). e)

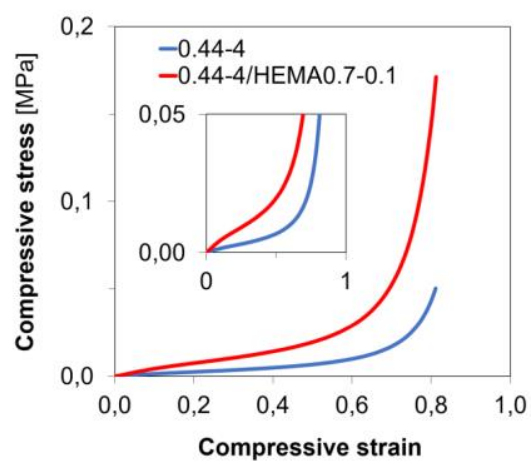

f)

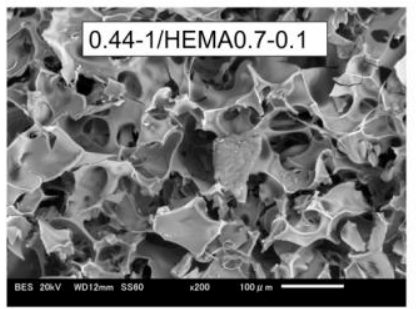

Figure 3. Mechanical properties and morphology of the TN cryogels (a-d) and DN cryogel with PHEMA as the 2nd network (e, f): The compressive test of TN cryogels (a) and moduli of TN cryogels plotted with moduli of the corresponding SN and DN cryogels (c, d). The morphology of freeze-dried TN cryogels as revealed by the SEM (b). The compressive test of DN cryogel with PHEMA 2nd network and its corresponding SN cryogel (e) and the morphology of such a DN cryogel (f).

SEM observation of the freeze-dried samples revealed that the original gross morphology of SN cryogels, with large pores $(\sim 50-100 \mu \mathrm{m})$ and large interconnections, was maintained after the $2^{\text {nd }}$ cryogelation in both the DN cryogel 0.44-4 and 0.44-1 series (Figure 1c, d). Such size of macropores and connectivity is suitable for cell cultivation and various tissue engineering applications. ${ }^{2-4}$ After the introduction of the $2^{\text {nd }}$ network, the sample dimensions only slightly increased by swelling. For example, the 0.44-4/0.70.1 sample diameter increased from $\sim 3.5 \mathrm{~mm}$ to $\sim 3.8 \mathrm{~mm}$, corresponding to an increase of $\sim 9 \%$ in pore size or $\sim 28 \%$ of their volume by volume of pore size. The equilibrium water regain (ratio $Q_{w}$ ) decreased with the second monomer concentration increase because of the increase in the polymer content (Figure 1e, f). $Q_{w}$ is the weight ratio of the swollen sample, including water present in both macropores and their gel walls, to the dry polymer. Based on the water squeezing experiment, the gel phase of DN gels that was based on SN 0.44-4 consisted of 26-34 wt.\% of the dry polymer and $\sim 66-74$ wt.\% of water, that was $\sim 13-17$ wt.\% of the total water (Figure S3). The porosity of the samples was slightly decreased (Figure 1e, f) by the formation of the $2^{\text {nd }}$ network, despite the SEM images showing that the gross morphology remained unchanged. The porosities of the DN cryogels based on the cyclohexane uptake were higher and decreased less steeply than those of the cryogels based on the water squeezing experiment, suggesting uneven shrinking of the gel phase during freeze-drying.

\subsection{Mechanical performance of DN cryogels}

The results of the compressive testing of SN cryogels and the corresponding DN cryogels are shown in Figure 2a and $\mathbf{3 b}$, respectively. For example, the compressive modulus of DN cryogels made from SN cryogel (sample 0.44-4) was about 2- to 4-times higher (depending on the concentration of $2^{\text {nd }}$ PAAm network) than the compressive modulus of the original SN cryogel (Figure 2 b). DN cryogels were also significantly tougher under compression than the 
corresponding SN cryogel, as shown by comparing the area under the compressive curves (Figure 2a). Typically, while stress at $60 \%$ compression reaches about $15 \mathrm{kPa}$ for SN cryogel $0.44-4$, it is more than $100 \mathrm{kPa}$ for the corresponding DN cryogel $0.44-4 / 1.4-0.1$. Higher $2^{\text {nd }}$ network concentration resulted in higher compressive modulus and tougher DN cryogels. Some of the previous studies on IPN cryogels have shown an increase in the modulus of porous gels, but that does not necessarily translate to a noticeable increase in strength and toughness. A similar trend was observed in DN cryogels prepared from SN cryogel 0.44-1 (Figure 2c, d) DN cryogels from this series are softer than those from the 0.44-4 series because of their lower amount of the $1^{\text {st }}$ network crosslinker. When no crosslinker was used during the formation of the $2^{\text {nd }}$ network, the prepared semi-IPN cryogels were less tough than DN cryogels (Figure S4).

The high strength of the prepared DN cryogels also enabled the characterization of the tensile behavior of these supermacroporous hydrogels. The uniaxial tensile behavior of the DN cryogels is depicted in Figure 2e. The stretching ability of DN cryogels was larger than that of the SN cryogels (the individual $1^{\text {st }}$ network and $2^{\text {nd }}$ network porous gels). In case of DN cryogels made of SN cryogel 0.44-1 with a lower amount of the crosslinker, the stretching ability of DN cryogels even exceeded that of PAAm cryogel (corresponding to $2^{\text {nd }}$ network), which was very soft. The stretching ability of DN cryogels was up to 2.2-times of the original sample length, depending on the composition of the DN cryogels. The tensile modulus and strength of DN cryogels increased with increasing concentration of PAAm $2^{\text {nd }}$ network. For example, the tensile modulus of the DN cryogel made from SN cryogel $0.44-4$ was up to 3-times higher than that of the corresponding SN cryogel ( $\sim 140 \mathrm{kPa}$ vs $\sim 50 \mathrm{kPa}$, Figure 2f). The tensile strength of DN cryogels was up to $\sim 200 \mathrm{kPa}$, which was two orders higher than that of SN cryogels.

The characteristic feature of DN gels is the large hysteresis upon cyclic loading. ${ }^{16}$ The larger hysteresis results in the dissipation of higher mechanical energy by rupture of $1^{\text {st }}$ network bonds, thereby resulting in high toughness of DN gel. Figure $2 \mathrm{~g}$ shows the results of the cyclic tensile test of DN cryogels, wherein the hysteresis is clearly visible. While the dissipated energy, that is the enclosed area of the hysteresis loop, is insignificant for sample 0.44-4/0.7-0.1, samples 0.44-4/1.4-0.1 and 0.44-1/0.7-0.1 show relatively large hysteresis because of the increase in the amount of the $2^{\text {nd }}$ network relative to the $1^{\text {st }}$ network. The work of extension $\left(\mathrm{kJ} \mathrm{m}^{-3}\right)$, which is the area below the stress-strain curve, was up to $38 \mathrm{~kJ} \mathrm{~m}^{-3}$ for DN cryogels (sample 0.44-4/1.4-0.1). In comparison, it was only $0.35 \mathrm{~kJ} \mathrm{~m}^{-3}$ for the corresponding SN cryogel (sample $0.44-4$ ) and $2.3 \mathrm{~kJ} \mathrm{~m}^{-3}$ for the PAAm cryogel (the corresponding $2^{\text {nd }}$ network gel). Therefore, it can be concluded that two types of tough DN cryogels can be prepared: 1) stiffer and less stretchable DN cryogels (sample 0.44-4/1.4-0.1) made from stiffer SN cryogel by using a higher concentration of $2^{\text {nd }}$ network pre-gel solution, and 2) softer but more stretchable DN cryogels (sample 0.441/0.7-0.1) made from softer SN cryogel by using a lower concentration of $2^{\text {nd }}$ network pre-gel solution. The existence of hysteresis distinguishes supermacroporous DN gels from other IPN gels. The supermacroporous hydrogels made from PNaAMPS and PAAm networks were, thus, proven to belong to DN gels.

3.3 TN cryogels and DN cryogels with different $2^{\text {nd }}$ network

DN cryogels $0.44-4 / 0.7-0.1,0.44-1 / 0.7-0.1$, and $0.44-$ 1/1.4-0.1 were used to prepare TN cryogels. Then, we investigated how the compressive toughness of TN cryogels can be increased by introducing a $3^{\text {rd }}$ network. The compressive test of TN cryogels is shown in Figure 3a. TN cryogels were tougher and stiffer than the corresponding DN cryogels under compression (compare Figure 2a, b with Figure 3a). The compressive modulus of the TN cryogels increased with increase in the $2^{\text {nd }}$ network concentration (Figure 3c, d). TN cryogels originating from SN cryogels with higher amount of the crosslinker (4 mol.\%, sample 0.44-4/0.7-0.1/0.7-0.1) were significantly stiffer than those originating from SN cryogel with the lower amount of the crosslinker (1 mol.\%, samples 0.44-1/0.7-0.1/0.7-0.1 and 0.44-1/1.4-0.1/1.4-0.1). The morphology of TN cryogel 0.44-4/0.7-0.1/0.7-0.1 was changed more than that of TN cryogel 0.44-1/0.7-0.1/0.7-0.1. However, both these samples still showed an open-cell morphology with large interconnected pores. The total concentration of $2^{\text {nd }}$ and $3^{\text {rd }}$ pregel solution was $1.4 \mathrm{~mol} \mathrm{~L}^{-1}$ for both samples, which is about 10 wt.\%. The morphology of TN cryogel 0.44-4/1.4-0.1/1.40.1 was considerably changed. This morphology change was attributed to the high total concentration of $2^{\text {nd }}$ and $3^{\text {rd }}$ pregel solution that was $2.8 \mathrm{~mol} \mathrm{~L}^{-1}$, about $20 \mathrm{wt}$. \%. The denser the DN cryogel's gel phase, the less likely the $3^{\text {rd }}$ network would be formed without affecting the morphology of DN cryogel.

Since poly(2-hydroxyethyl methacrylate) (PHEMA) and poly $(N, N$-dimethylacrylamide) (PDMAAm) are biocompatible polymers, we prepared DN cryogels, using HEMA or DMAAm as the monomer for creation of the $2^{\text {nd }}$ network, by the same procedure replacing AAm monomer. The DN cryogel 0.44-4/HEMA0.7-0.1 with the PHEMA $2^{\text {nd }}$ network also showed higher modulus and toughness under compression than the original SN cryogel 0.44-4 (Figure 3e). The modulus of this DN cryogel with $2^{\text {nd }}$ PHEMA network increased three times from $13.2 \pm 2.7 \mathrm{kPa}$ to $40.2 \pm 6.7 \mathrm{kPa}$ compared to that of the original SN cryogel. Meanwhile, the original supermacroporous morphology of the SN cryogel was maintained, as shown in Figure 3f. However, when DMAAm was used for the formation of the $2^{\text {nd }}$ network, the cryogels became stiffer, but the morphology was significantly changed, probably due to the more hydrophobic nature of DMAAm (Figure S5). It seems that DMAAm polymerizes mostly in pores of SN cryogel. It is because most of DMAAm does not diffuse into the gel phase of SN cryogel. Moreover, hydrophobicity of DMMAm can lead to different crystallization rate and, hence, to different size of ice crystals; change in $2^{\text {nd }}$ cryogelation temperature could eventually lead to better results. Nevertheless, the DN cryogels with the PDMAAm $2^{\text {nd }}$ network still showed an open-cell morphology. Therefore, these results indicate that, for the preparation of supermacroporous DN hydrogels that are based on 
highly hydrophilic $1^{\text {st }}$ network, the two-step cryogelation technique should be used preferably with hydrophilic than with hydrophobic monomers to form the $2^{\text {nd }}$ network.

The question why modulus of DN cryogels is two orders lower than that of non-porous DN hydrogels can be simply explained adopting the following equation ${ }^{5}$ :

$$
E=E_{0} \cdot(1-P)^{2}
$$

where $E_{0}$ is Young's modulus of non-porous hydrogel, $E$ is Young's modulus of respective porous hydrogel with the same composition, and $P$ is the volume fraction of pores in the porous hydrogel. Similar relation to the dependence of material strength on porosity can be found in the literature. ${ }^{6}$ The porosity of our DN cryogels ranged between 71 to $85 \%$ determined by gel squeezing experiment (Figure 1e, f). Despite the lower compressive and tensile moduli of supermacroporous DN hydrogels in comparison to non-porous DN hydrogels, the mechanical performance of these materials far exceeds these of original supermacroporous SN hydrogels. The developed method for preparation of DN cryogels represents original instructions on how to prepare tough supermacroporous hydrogels.

\subsection{Structural analysis of DN cryogels}

To observe the structure of the samples in the pristine swollen state, DN cryogel 0.44-4/0.7-0.1 was stained with fluorescent dyes and subjected to visualization by confocal laser scanning microscopy (CLSM; Figure 4a-c). The images of PNaAMPS $1^{\text {st }}$ network, that was stained with Rhodamine B (red fluorescence), and PAAm $2^{\text {nd }}$ network, that was stained by copolymerization with $o$-fluorescein acrylate (green fluorescence), showed perfect overlap, confirming that the $2^{\text {nd }}$ network was polymerized mainly in the $1^{\text {st }}$ network phase and not in the pores. The DN cryogel $0.44 / 0.7$ 0.1 was also treated with a $\mathrm{PbNO}_{3}$ solution, which allowed visualization of the swollen sample by nano-CT with enough contrast (Figure 4d-f). The nano-CT enabled advanced 3D analysis of the sample structure. The average distance of gel walls was calculated as $52.9 \pm 15.8 \mu \mathrm{m}$. The gel contained mostly highly connected spherical pores $40-70 \mu \mathrm{m}$ in diameter and longitudinal pores of up to approximately $230 \mu \mathrm{m}$ long. The size of connections between pores was $23 \pm 10 \mu \mathrm{m}$ in diameter. Such morphology is sufficient for migration of various human cells. ${ }^{3,4}$ The thicknesses distribution of the macropores gel walls was calculated as $11.3 \pm 4.8 \mu \mathrm{m}$. The gel wall thickness was up to $27 \mu \mathrm{m}$ at the gel nodes (crosspoints of gel walls). 

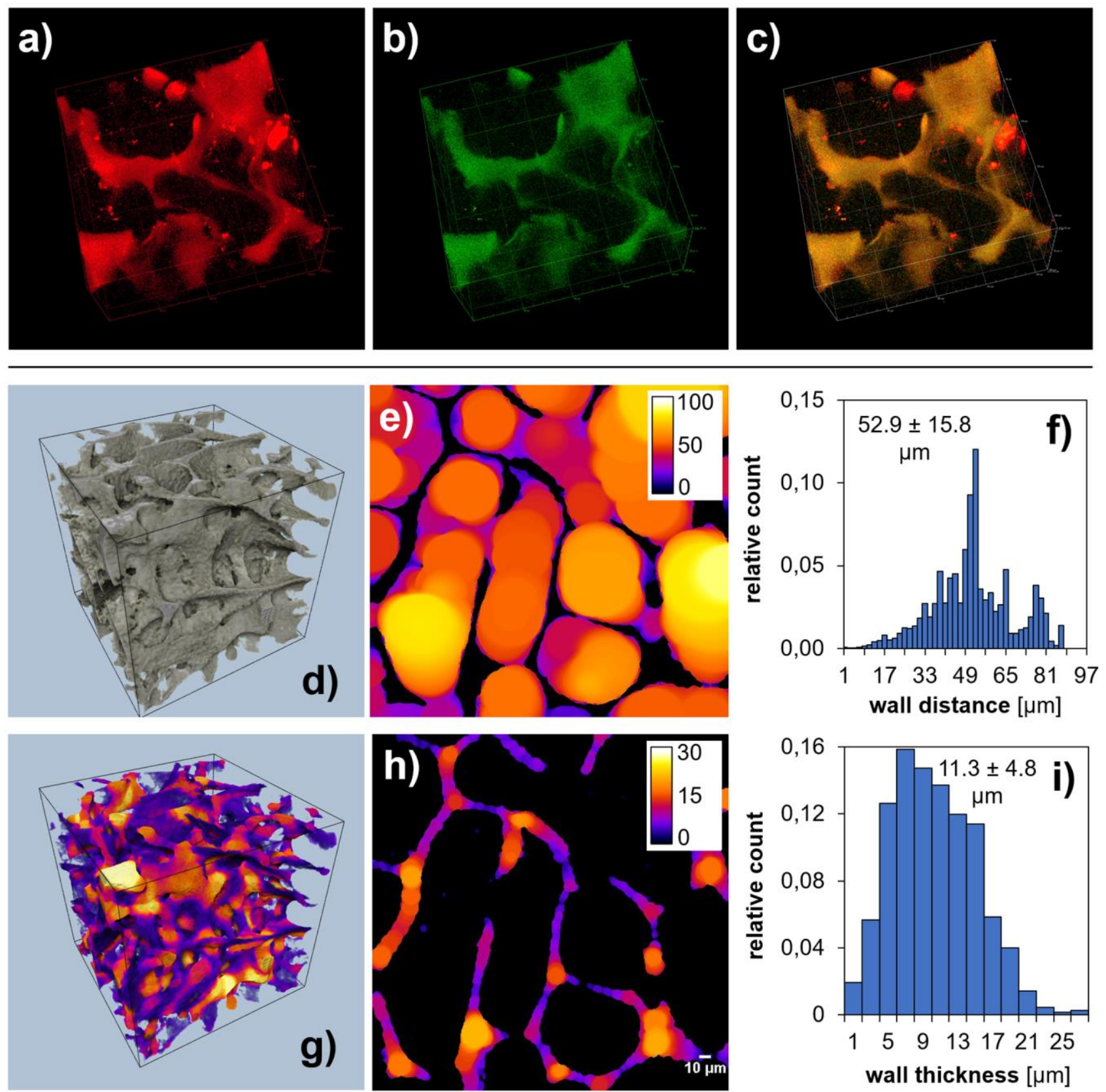

Figure 4. Visualization of swollen DN cryogel 0.44-4/0.7-0.1 by CLSM (a-c), the element size is $\left.(210 \mu \mathrm{m})^{2} \times 100 \mu \mathrm{m}\right)$ and by nano-CT with 3D analysis (d-i). For CLSM, PNaAMPS $1^{\text {st }}$ network was stained with Rhodamine B, red fluorescence (a), PAAm $2^{\text {nd }}$ network was stained by copolymerization with $o$-fluorescein acrylate, green fluorescence (b), merged CLSM images (c); DN cryogel for nano-CT was stained through $1^{\text {st }}$ network by $\mathrm{PbNO}_{3}$ solution: reconstructed $3 \mathrm{D}$ image, the element size is $\left.(300 \mu \mathrm{m})^{3}\right)(\mathrm{d})$, the wall distance $(\mathrm{e}, \mathrm{f})$ and the wall thickness 3D analyzes (g-i), where the colors in individual scans represent the wall distance and wall thickness, respectively. The wall distance and wall thickness distributions (f, i).

\section{Conclusions}

In this work, we established a two-step cryogelation technique to produce tough supermacroporous hydrogels, with opencell morphology, based on the DN strategy. This technique enabled the synthesis of the $2^{\text {nd }}$ network while preserving the porous structure of the $1^{\text {st }}$ network gel. When the $2^{\text {nd }}$ pre-gel solution was frozen under certain condition, ice crystallization occurred preferentially in the pores, permitting the synthesis of the $2^{\text {nd }}$ network while maintaining the porous structure of the $1^{\text {st }}$ network gel. We showed that original open-cell morphology of the $1^{\text {st }}$ network cryogels can be well preserved when 
the $2^{\text {nd }}$ and even $3^{\text {rd }}$ networks are introduced. We also demonstrated that the compressive and tensile properties of the brittle supermacroporous SN hydrogels can be significantly enhanced by using the DN/TN strategy. The high toughness distinguishes the DN cryogels from conventional IPN cryogels. Interestingly, DN cryogels also showed improved cutting resistance. In addition, the semi-transparency of some DN cryogels could still provide an advantage for the observation of cell behavior during cultivation. Furthermore, we assume that while the proposed technique could be used universally for the preparation of tough supermacroporous hydrogels by cryogelation, the exact conditions differ for different gel precursors. To the best of our knowledge, DN cryogels based on PNaAMPS and PAAm prepared in this study are the toughest cryogels with open-cell morphology reported. These DN cryogels will meet the high demand on stiffer and stronger supermacroporous hydrogels for advanced load-bearing applications, including the biomedical field.

\section{ASSOCIATED CONTENT}

Supporting Information. The video showing the mechanical weakness of the pure first and second SN cryogels and the significant enhancement of the mechanical properties of the corresponding DN cryogel. A detailed description of the SN cryogels, including the compressive test and morphology revealed by SEM, analysis of equilibrium water content of the DN cryogels gel phase, comparison of the mechanical properties of DN cryogels to corresponding semi-IPN cryogels, and compressive test of DN cryogels with PDMAAm $2^{\text {nd }}$ network. This material is available free of charge via the Internet at http://pubs.acs.org.

\section{AUTHOR INFORMATION}

\section{Corresponding Author}

* Prof. Jian Ping Gong, e-mail: gong@sci.hokudai.ac.jp

\section{Author Contributions}

The manuscript was written through contributions of all authors. / All authors have given approval to the final version of the manuscript.

\section{ACKNOWLEDGMENT}

The authors thank Dr. Y. Katsuyama, and Ms. Y. Hane for their help with experiments. This work was supported by the Japan Society for the Promotion of Science (JSPS) KAKENHI (Grant Number 17H06144, JP17H06376). JPG acknowledges the Institute for Chemical Reaction Design and Discovery (WPI-ICReDD), that was established by the World Premier International Research Initiative (WPI), MEXT, Japan. We would like to thank Editage (www.editage.com) for English language editing.

\section{ABBREVIATIONS}

AAm, acrylamide; APS, ammonium persulfate; BSE, backscattered electrons; CLSM, confocal laser scanning microscopy; DMAAm, $N, N$-dimethylacrylamide; DN, double-network; HEMA, 2-hydroxyethyl methacrylate; IPN, interpenetrating networks, MBAAm, $N, N^{\prime}-$ methylenebis(acrylamide); NaAMPS, 2-acrylamido-2-methylpropane sulfonic acid, sodium salt; nano-CT, nanotomography; 2-oxo, 2-oxoglutaric acid; PAAm, polyacrylamide; PDMAAm, poly(N,N-dimethylacrylamide), PHEMA, poly(2-hydroxyethyl methacrylate); PNaAMPS, sodium poly(2-acrylamido-2-methylpropane sulfonate); SEM, scanning electron microscopy, SN, single-network; TEMED, $N, N, N^{\prime}, N^{\prime}$-tetramethylenediamine; NaAMPS; TN, tripe-network. 


\section{REFERENCES}

[1] Kumar, A. In Supermacroporous Cryogels: Biomedical and Biotechnological Applications; Kumar, A., Ed.; CRC Press: Boca Raton, FL, 2016; pp ix.

[2] IUPAC. Compendium of Chemical Terminology, $2^{\text {nd }}$ ed. (the "Gold Book"). Compiled by McNaught, A. D.; Wilkinson, A. Blackwell Scientific Publications, Oxford (1997). Online version (2019) created by Chalk, S. J. ISBN 0-9678550-9-8. https://doi.org/10.1351/goldbook.

[3] Annabi, N.; Nichol, J. W.; Zhong, X.; Koshy, Ch.; Ji, S.; Khademhosseini, A.; Dehghani, F. Controlling the porosity and microarchitecture of hydrogels for tissue engineering. Tissue Eng, Part B 2010, 16, 371-383.

[4] Whang, K.; Healy, K. E.; Elenz, D. R.; Nam, E. K.; Tsai, D. C.; Thomas, C. H.; Nuber, G. W.; Glorieux, F. H.; Travers, R.; Sprague, S. M. Engineering Bone Regeneration with Bioabsorbable Scaffolds with Novel Microarchitecture. Tissue Eng. 1999, 5, 35-51.

[5] Welzel, P. B.; Friedrichs, J.; Grimmer, M.; Vogler, S.; Freudenberg, U.; Werner, C. Cryogel Micromechanics Unraveled by Atomic Force Microscopy-Based Nanoindentation. Adv. Healthc. Mater. 2014, 3, 1849-1853.

[6] LaNasa, S. M.; Hoffecker, I. T.; Bryant, S. J. Presence of pores and hydrogel composition influence tensile properties of scaffolds fabricated from well-defined sphere templates. J. Biomed. Mater. Res. B: Appl. Biomater. 2010, 96B, 294-302.

[7] Pippan, R.; Wurster, S.; Kiener, D. Fracture mechanics of micro samples: Fundamental considerations. Mat. Des. $2018,159,252$.

[8] Sedlačík, T.; Proks, V.; Šlouf, M.; Dušková-Smrčková, M.; Studenovská, H.; Rypáček, F. Macroporous Biodegradable Cryogels of Synthetic Poly( $\alpha$-amino acids). Biomacromolecules 2015, 16, 3455-3465.

[9] Ingavle, G. C.; Baillie, L. W. J.; Zheng, Y.; Lis, E. K.; Savina, I. N.; Howell, C. A.; Mikhalovsky, S. V.; Sandeman, S. R. Affinity binding of antibodies to supermacroporous cryogel adsorbents with immobilized protein A for removal of anthrax toxin protective antigen. Biomaterials 2015, 50, 140-153.

[10] Gong, J.; Katsuyama, Y.; Kurokawa, T.; Osada, Y. Double-Network Hydrogels with Extremely High Mechanical Strength. Adv. Mater. 2003, 15: 1155-1158

[11] Sun, J.; Zhao, X.; Illeperuma, W.; Chaudhuri, O.; Oh, K. H.; Mooney, D. J.; Vlassak, J. J.; Suo, Z. Highly stretchable and tough hydrogels. Nature 2012, 489, 133-136.

[12] Imran, B. A.; Esaki, K.; Gotoh, H.; Seki, T.; Ito, K.; Sakai, Y.; Takeoka, Y. Extremely stretchable thermosensitive hydrogels by introducing slide-ring polyrotaxane cross-linkers and ionic groups into the polymer network. Nat. Commun. 2014, 5, 5124.

[13] Xu, L.; Wang, Ch.; Cui, Y.; Li, A.; Qiao, Y.; Qiu, D. Conjoined-network rendered stiff and tough hydrogels from biogenic molecules. Science Advances 2019, 5, eaau3442.

[14] Grijalvo, S.; Eritja, R.; Díaz, D. D. On the Race for More Stretchable and Tough Hydrogels. Gels 2019, 5, 24.

[15] Liu, Y.; He, W.; Zhang, Z.; Lee, B. P. (2018). Recent Developments in Tough Hydrogels for Biomedical Applications. Gels 4, 46.

[16] Gong, J. P. Why are double network hydrogels so tough? Soft Matter 2010, 6, 2583-2590.

[17] Chen, Q.; Chen, H.; Zhu, L.; Zheng, J. Fundamentals of double network hydrogels. J. Mater. Chem. B 2015, 3, 3654-3676.

[18] Haque, M. A.; Kurokawa, T.; Gong, J. P. Super tough double network hydrogels and their application as biomaterials. Polymer 2012, $53,1805-1822$.

[19] Nonoyama, T.; Wada, S.; Kiyama, R.; Kitamura, N.; Mredha, M. T. I.; Zhang, X.; Kurokawa, T.; Nakajima, T.; Takagi, Y.; Yasuda, K.; Gong, J. P. Double-Network Hydrogels Strongly Bondable to Bones by Spontaneous Osteogenesis Penetration. Adv. Mater. 2016, 28, 67406745 .

[20] Kovačič, S.; Silverstein, M. S. Hydrogels through emulsion templating: sequential polymerization and double networks. Polym. Chem. 2017, 8, 6319-6328.

[21] Lozinsky V. I. Cryostructuring of Polymeric Systems. 50. Cryogels and Cryotropic Gel-Formation: Terms and Definitions. Gels 2018, $4,77$.

[22] Lozinsky, V. I.; Okay, O. In Advances in Polymer Science, Vol. 263; O. Okay, O., Ed.; Springer International Publishing: Cham, Switzerland, 2014; pp. 49-101.

[23] Henderson, T. M. A.; Ladewig, K.; Haylock, D. N.; McLean, K. M.; O’Connor, A. J. Cryogels for biomedical applications. J. Mater. Chem. B 2013, 1, 2682-2695.

[24] Hixon, K. R.; Lu, T.; Sell, S. A. A comprehensive review of cryogels and their roles in tissue engineering applications. Acta Biomater. $2017,62,29-41$.

[25] Memic, A.; Colombani, T.; Eggermont, L. J.; Rezaeeyazdi, M.; Steingold, J.; Rogers, Z. J.; Navare, K. J.; Mohammed, H. S.; Bencherif, S. A. Latest Advances in Cryogel Technology for Biomedical Applications. Adv. Therap. 2019, 1800114.

[26] Plieva, F. M.; Ekström, P.; Galaeva, I. Y.; Mattiasson, B. Monolithic cryogels with open porous structure and unique double-continuous macroporous networks. Soft Matter 2008, 4, 2418-2428.

[27] Yetiskin, B.; Akinci, C.; Oguz Okay. Cryogelation within cryogels: Silk fibroin scaffolds with single-, double- and triple-network structures. Polymer 2017, 128, 47-56.

[28] Jain, E.; Kumar, A. Designing supermacroporous cryogels based on polyacrylonitrile and a polyacrylamide-chitosan semi-interpenetrating network. J. Biomater. Sci. Polym. Ed. 2009, 20, 877-902.

[29] Jain, E.; Srivastava, A; Kumar, A. Macroporous interpenetrating cryogel network of poly(acrylonitrile) and gelatin for biomedical applications. J. Mater. Sci.: Mater. Med. 2009, 20, S173-S179.

[30] Kathuria, N.; Tripathi, A.; Kar, K.; Kumar, A. Synthesis and characterization of elastic and macroporous chitosan-gelatin cryogels for tissue engineering. Acta Biomater. 2009, 5, 406-418.

[31] Bhat, S.; Tripathi, A.; Kumar, A. Supermacroprous chitosan-agarose-gelatin cryogels: in vitro characterization and in vivo assessment for cartilage tissue engineering. J. R. Soc. Interface 2011, 8, 540-554.

[32] Zhao, Q.; Sun, J.; Wua, X.; Lin, Y. Macroporous double-network cryogels: formation mechanism, enhanced mechanical strength and temperature/pH dual sensitivity. Soft Matter 2011,7, 4284-4293.

[33] Dragan, E. S.; Lazar, M. M.; Dinu, M. V.; Doroftei, F. Macroporous composite IPN hydrogels based on poly(acrylamide) and chitosan with tuned swelling and sorption of cationic dyes. Chem. Eng. J. 2012, 204-206, 198-209. 
[34] Dragan, E. S.; Loghin, D. F. A.; Cocarta, A.-I.; Doroftei, M. Multi-stimuli-responsive semi-IPN cryogels with native and anionic potato starch entrapped in poly(N,N-dimethylaminoethyl methacrylate) matrix and their potential in drug delivery. React. Funct. Polym. 2016, 105, 66-77.

[35] Meena, L. K.; Raval, P.; Kedaria, D.; Vasita, R. Study of locust bean gum reinforced cyst-chitosan and oxidized dextran based semiIPN cryogel dressing for hemostatic application. Bioact. Mater. 2018, 3, 370-384.

[36] Shen, Z.-S.; Cui, X.; Hou, R.-X.; Li, Q.; Deng, H.-X.; Fu, J. Tough biodegradable chitosan-gelatin hydrogels via in situ precipitation for potential cartilage tissue engineering. RSC Adv. 2015, 5, 55640-55647.

[37] Tripathi, A.; Kathuria, N.; Kumar, A. Elastic and macroporous agarose-gelatin cryogels with isotropic and anisotropic porosity for tissue engineering. J. Biomed. Mater. Res. 2009, 90A, 680-694.

[38] Plieva, F. M.; Kumar, A.; Galaev, Y. I.; Mattiasson, B. In Advanced Biomaterials: Fundamentals, Processing and Applications, $1^{\text {st }}$ ed.; Basu, B.; Katti, D. S.; Kumar, A., Eds.; John Wiley \& Sons, Inc.: Hoboken, NJ, 2009; Chapter 14, pp 499-534.

[39] Caglio, S.; Righetti, P. G. On the pH dependence of polymerization efficiency, as investigated by capillary zone electrophoresis. Electrophoresis 1993, 14, 554-558.

[40] Limaye, A. Drishti: a volume exploration and presentation tool. Proc. IEEE 2012, 8506, 85060X.

[41] Schneider, C. A.; Rasband, W. S.; Eliceiri, K. W. NIH Image to ImageJ: 25 Years of Image Analysis. Nat. Methods 2012, 9, $671-675$.

[42] Hildebrand, T.; Rüegsegger, P. A new method for the model-independent assessment of thickness in three-dimensional images. J. Microsc. 1996, 185, 67-75. 
SYNOPSIS TOC. Supermacroporous double-network (DN) hydrogels are prepared by a two-step cryogelation, that is cross-linking polymerization in a frozen state where ice crystals act as porogens. Although the $1^{\text {st }}$ and $2^{\text {nd }}$ single-network (SN) cryogels are weak, DN cryogels showed synergistically improved mechanical properties. Cryogelation is also conducted to prepare even tougher triple-network (TN) cryogels.

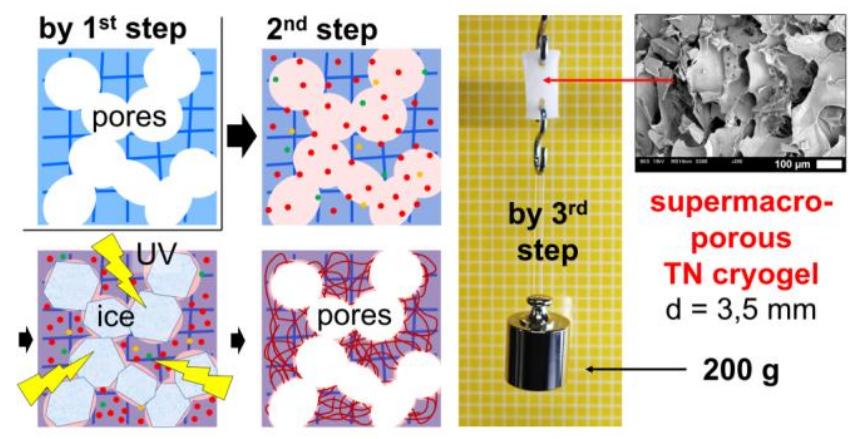

